\title{
Radical Cyclocopolymerization of Divinyl Ether Derivatives with Maleic Anhydride. The Structure Determination of the Alternating Copolymers by ${ }^{13} \mathrm{C}-\mathrm{NMR}$ Spectroscopy*
}

\author{
Mitsuo TsUKINO and Toyoki KUnITAKE** \\ Department of Chemical Engineering, Kitakyushu Technical College, \\ Kokura-minami, Kitakyushu 803 and Department of Organic Synthesis, \\ Faculty of Engineering, Kyushu University, Fukuoka 812 Japan.
}

(Received November 29, 1980)

\begin{abstract}
Radical copolymerizations of cis-propenyl vinyl ether, 2-methylpropenyl vinyl ether and cis-dipropenyl ether with maleic anhydride were carried out, and completely cyclized, and alternating copolymers were obtained with the $1: 2$ composition of divinyl ethers and maleic anhydride. ${ }^{13} \mathrm{C}$-NMR spectroscopic data indicated that the polymers contained in common the bicyclic unit composed of one molecule of each monomer and the maleic anhydride unit. The bicyclic unit was characterized by the cis junction and the trans ring closure, and the maleic anhydride unit was formed by trans opening of the double bond. Introduction of methyl substituents do not appear to affect the polymer structure, though assignments become less definite.

KEY WORDS ${ }^{13}$ C-NMR Spectroscopy / Cyclopolymerization / Divinyl Ethers / Maleic Anhydride / Alternating Copolymer /
\end{abstract}

The cyclocopolymer of divinyl ether and maleic anhydride is attracting much attention, since its hydrolyzed product possesses various biological activities such as anti-tumor activity, interferon induction, antibacterial activity, immune response stimulation, etc. ${ }^{1,2}$

Although the formation of the cyclocopolymer has been known for some time, a definite structure determination of the polymer was not made until recently. In his pioneering study, Butler ${ }^{3}$ proposed the presence of the $[4,3,0]$ bicyclic structure as in $\mathbf{I}$.

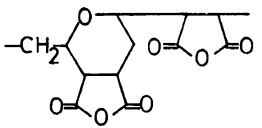

I

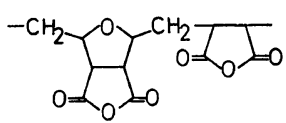

II
Butler and $\mathrm{Chu}^{4}$ reconfirmed this structure by their recent $300 \mathrm{MHz}{ }^{1} \mathrm{H}-\mathrm{NMR}$ study of the partially

* Contribution No. 612 from Department of Organic Synthesis.

** Correspondence should be setn to this author at the Fukuoka address.

deuterated polymer. On the other hand, Samuels ${ }^{5}$ suspected the formation of the $[3,3,0]$ bicyclic unit in II, from polymer solution measurements and molecular model studies.

We investigated the structure of the $1: 2$ cyclocopolymer by ${ }^{13} \mathrm{C}$-NMR spectroscopy and concluded that the polymer was composed predominantly of structure $\mathbf{H I}^{6}{ }^{6}$ The steric structure of the copolymer could be also determined by a comparison of the observed and calculated chemical shifts.

In this paper, we extended our previous work to divinyl ether derivatives, and examined the polymer structures by ${ }^{13} \mathrm{C}-\mathrm{NMR}$ spectroscopy. The divinyl monomers used are as follows.<smiles>C=COC=C</smiles>

Divinyl ether (DVE)<smiles>C=COC=C(C)C</smiles>

2-Methylpropenyl vinyl cis-Dipropenyl ether (DPE) ether $\left(\mathrm{CH}_{3}-\mathrm{PVE}\right)$ 


\section{EXPERIMENTAL}

\section{Materials}

The preparation and purification of divinyl ether (DVE), cis-propenyl vinyl ether (PVE) and 2methylpropenyl vinyl ether $\left(\mathrm{CH}_{3}-\mathrm{PVE}\right)$ are described elsewhere. ${ }^{7.8}$ cis-Dipropenyl ether (DPE) was prepared by the base-catalyzed rearrangement of diallyl ether. Diallyl ether (bp $94-95^{\circ} \mathrm{C}$, lit ${ }^{9}$ $94^{\circ} \mathrm{C}$ ) was treated by potassium tert-butoxide in dimethylsulfoxide at $90^{\circ} \mathrm{C}$ for $90 \mathrm{~h}$. The isomerized product was distilled, washed with alkali and water, and redistilled: bp $90-92^{\circ} \mathrm{C}\left(1 \mathrm{lit}^{10} 90-94^{\circ} \mathrm{C}\right), d_{4}^{25}$ 0.8016 . Gas chromatography and IR and NMR spectroscopies indicated that the product contained $3-4 \%$ of the cis,trans- or trans,trans-isomer as the sole impurity.

$\alpha, \beta$-Diethylsuccinic anhydride ${ }^{11}$ was prepared by refluxing thionyl chloride and $\alpha, \beta$-diethylsuccinic acid ${ }^{12}$ obtained by the acid $\left(\mathrm{H}_{2} \mathrm{SO}_{4}\right)$ hydrolysis of ethyl $\alpha$-cyano- $\alpha, \beta$-diethylsuccinate. ${ }^{13}$ The product was characterized by elemental analysis and IR and NMR spectroscopies. ${ }^{13} \mathrm{C}-\mathrm{NMR}$ data of the product is shown below.<smiles>CCC1C(=O)OC(=O)C1[14CH2][14CH3]</smiles><smiles>CCC1C(=O)OC(=O)C1[14CH2]C</smiles>

Maleic anhydride (MAn) was purified by sublimation and azobisisobutyronitrile (AIBN) was recrystallized from ethanol. Solvents were purified by the usual procedure.

\section{Polymerization}

Given amounts of monomers, AIBN and solvent were placed in ampoules and subjected to the freezepump-thaw cycle several times. The ampoules were then sealed in vacuo and immersed in a constant temperature bath. The polymers obtained in dimethylformamide (DMF) and acetone were precipitated in ether and reprecipitated from acetone and ether. In other solvents, the polymers precipitated during polymerization were collected and reprecipitated from acetone and ether. The purified polymers were dried in vacuo at room temperature for $15 \mathrm{~h}$. The copolymer composition was estimated by elemental analysis after correction for the water content. The water content was $4-5 \%$ for all the polymers.

\section{Instruments}

The measurement of ${ }^{13} \mathrm{C}$-NMR spectra was conducted with a Bruker WH-90 FT-NMR instrument (22.63 MHz) under noise decoupling. ${ }^{1} \mathrm{H}-\mathrm{NMR}$ and IR spectra were obtained with Varian A60 $(60 \mathrm{MHz})$ and Shimadzu IR-400 instruments, respectively. UV spectra were obtained with a Shimadzu UV-400 instrument.

\section{RESULTS AND DISCUSSION}

\section{Formation of Charge-Transfer Complexes between Monomers}

When PVE and MAn were mixed in benzene, a pale yellow color developed. This was conceivably due to the formation of a charge-transfer complex. The role of charge-transfer complexes in the radical cyclopolymerization has been discussed by Butler and coworkers in detail. For instance, Butler and Fujimori ${ }^{14}$ observed a charge-transfer band between DVE and MAn at $270 \mathrm{~nm}$ and found a correlation between the equilibrium constant of the complex formation and the rate of copolymerization. Discoloration was more evident in the case of $\mathrm{CH}_{3}-\mathrm{PVE}$ and DPE. Figure 1 shows UV spectra of divinyl ether derivatives mixed with MAn. The position of $\lambda_{\max }$ shifts to longer wavelengths with increasing methyl substitution:

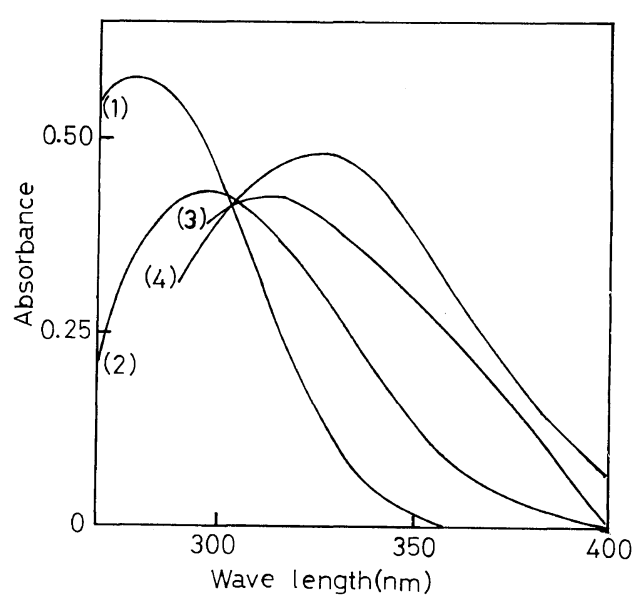

Figure 1. Electronic spectra of charge-transfer complexes of divinyl ethers with maleic anhydride: solvent, $\mathrm{CHCl}_{3}$; divinyl ethers, $1.0 \mathrm{~mol} \mathrm{l}^{-1}$; MAn, $7.5 \times 10^{-3} \mathrm{~mol}$ $1^{-1}$. Absorbances due to divinyl ethers and maleic anhydride are deducted.

(1), DVE+MAn; (2), PVE + MAn; (3) $\mathrm{CH}_{3}-\mathrm{PVE}+$ MAn; (4) DPE+MAn. 
Copolymerization of Divinyl Ethers and Maleic Anhydride

Table I. Radical copolymerization of PVE $\left(\mathrm{M}_{1}\right)$ and MAn $\left(\mathrm{M}_{2}\right)^{\mathrm{a}}$

\begin{tabular}{|c|c|c|c|c|c|c|c|}
\hline \multirow{2}{*}{ Run } & \multirow{2}{*}{$\begin{array}{c}\mathrm{M}_{2} \\
\text { Mole } \\
\text { fraction }\end{array}$} & \multirow{2}{*}{$\frac{\text { Total monomer. }}{\operatorname{mol~l}^{-1}}$} & \multirow{2}{*}{$\frac{\mathrm{AIBN}}{\mathrm{mol} \mathrm{1}^{-1}}$} & \multirow{2}{*}{$\frac{\text { Polymerization time }}{\min }$} & \multirow{2}{*}{$\frac{\text { Conversion }}{\%}$} & \multirow{2}{*}{$\begin{array}{c}\mathrm{m}_{2} \text { (Polymer) } \\
\text { mole } \\
\text { fraction }^{\mathrm{b}}\end{array}$} & \multirow{2}{*}[\eta]{$^{\mathrm{c}}$} \\
\hline & & & & & & & \\
\hline 1 & 0.15 & 2.00 & $5.0 \times 10^{-3}$ & 13 & 9.5 & 0.65 & \\
\hline 2 & 0.30 & 2.00 & $5.0 \times 10^{-3}$ & 13 & 9.4 & 0.65 & \\
\hline 3 & 0.50 & 2.00 & $5.0 \times 10^{-3}$ & 13 & 11.2 & 0.66 & \\
\hline 4 & 0.67 & 2.00 & $5.0 \times 10^{-3}$ & 12 & 9.6 & 0.66 & \\
\hline 5 & 0.75 & 2.00 & $5.0 \times 10^{-3}$ & 12 & 7.3 & 0.66 & \\
\hline 6 & 0.90 & 2.00 & $5.0 \times 10^{-3}$ & 20 & 6.0 & 0.67 & \\
\hline 7 & 0.33 & 2.00 & $1.0 \times 10^{-2}$ & 25 & 40.0 & 0.67 & \\
\hline 8 & 0.50 & 2.00 & $1.0 \times 10^{-2}$ & 25 & 43.7 & 0.66 & \\
\hline 9 & 0.67 & 2.00 & $1.0 \times 10^{-2}$ & 25 & 36.3 & 0.67 & \\
\hline 10 & 0.67 & 2.00 & $5.0 \times 10^{-3}$ & 20 & 60.5 & & 1.01 \\
\hline $11^{\mathrm{d}}$ & 0.67 & 0.60 & $2.5 \times 10^{-3}$ & 1290 & 82.7 & & 0.20 \\
\hline $12^{\mathrm{e}}$ & 0.67 & 2.00 & $1.0 \times 10^{-2}$ & 180 & 86.1 & 0.65 & 0.43 \\
\hline
\end{tabular}

a Polymerization conditions; $60^{\circ} \mathrm{C}$, benzene medium unless stated otherwise.

b Estimated from elemental analysis. $\mathrm{H}_{2} \mathrm{O}$ content was corrected.

c Measured in acetone at $30^{\circ} \mathrm{C}$.

d Polymerization solvent, acetone and $\mathrm{CS}_{2}\left(\mathrm{CS}_{2} 80 \mathrm{vol} \%\right)$.

e Polymerization solvent, acetone.

Table II. Radical compolymerization of $\mathrm{CH}_{3}-\operatorname{PVE}\left(\mathrm{M}_{1}\right)$ and DPE $\left(\mathrm{M}_{1}\right)$ with MAn $\left(\mathrm{M}_{2}\right)^{\mathrm{a}}$

\begin{tabular}{|c|c|c|c|c|c|c|c|c|}
\hline \multirow{3}{*}{$\begin{array}{l}\text { Run } \\
\text { No }\end{array}$} & \multirow{3}{*}{$\mathbf{M}_{1}$} & \multirow{3}{*}{$\begin{array}{c}\mathrm{M}_{1} \\
\text { Mole } \\
\text { fraction }\end{array}$} & \multirow{3}{*}{$\begin{array}{c}\begin{array}{c}\text { Total } \\
\text { monomer }\end{array} \\
\text { mol l-1 }^{-1}\end{array}$} & \multirow[t]{3}{*}{ Solvent } & \multirow{3}{*}{$\begin{array}{c}\text { Polymer- } \\
\text { ization } \\
\text { time }\end{array}$} & \multirow{3}{*}{$\frac{\text { Converstion }}{\%}$} & \multirow{3}{*}{$\begin{array}{l}\mathrm{m}_{2} \text { (Polymer) } \\
\text { mole fraction }\end{array}$} & \multirow{3}{*}[\eta]{$^{\mathrm{c}}$} \\
\hline & & & & & & & & \\
\hline & & & & & & & & \\
\hline 13 & $\mathrm{CH}_{3}-\mathrm{PVE}$ & 0.50 & 2.00 & DMF & 2.5 & 7.1 & & 0.07 \\
\hline 14 & $\mathrm{CH}_{3}-\mathrm{PVE}$ & 0.50 & 2.00 & $\mathrm{C}_{6} \mathrm{H}_{6}$ & 2.2 & 42.4 & 0.68 & \\
\hline 15 & $\mathrm{CH}_{3}-\mathrm{PVE}$ & 0.67 & 1.00 & $\mathrm{C}_{6} \mathrm{H}_{6}$ & 1.5 & 27.6 & 0.65 & \\
\hline 16 & DPE & 0.65 & 2.00 & $\mathrm{C}_{6} \mathrm{H}_{6}$ & 2.0 & 26.4 & 0.66 & 0.29 \\
\hline
\end{tabular}

a $60^{\circ} \mathrm{C}$; AIBN, $5.0 \times 10^{-3} \mathrm{~mol} \mathrm{1}^{-1}$.

b Estimated from elemental analysis. $\mathrm{H}_{2} \mathrm{O}$ content is corrected.

c Measured in acetone at $30^{\circ} \mathrm{C}$.

$\lambda_{\max }=282$ (DVE), 298 (PVE), $315\left(\mathrm{CH}_{3}-\mathrm{PVE}\right)$ and $326 \mathrm{~nm}$ (DPE).

The charge-transfer absorption was less apparent in polar media, as already noted for the DVE-MAn combination. ${ }^{14}$

\section{Copolymerization of PVE with MAn}

The copolymerization data of PVE and MAn are given in Table $I$. The copolymer composition (PVE: MAn unit) was always $1: 2$, as determined by elemental analysis after correction for water. This was confirmed by back titration of the polymer carboxylate for several samples. The copolymerization proceeded without gelation up to high conversions. Gelation occurred much more readily in the case of DVE-MAn. The copolymerization rate was fairly constant when the mole fraction of PVE was greater than $c a .30 \%$ (Table I, runs $1-4$ ). The specific viscosity of the copolymer was largest at the PVE fraction of $c a .70 \%$. Both the polymerization rate and the specific viscosity of the copolymer were smaller than $\mathrm{CS}_{2}$ or acetone used as the polymerization solvent in place of benzene (Table I, runs 11, 12). 
Copolymerization of $\mathrm{CH}_{3}-\mathrm{PVE}$ and DPE with $\mathrm{MAn}$

Table II summarizes the copolymerization of $\mathrm{CH}_{3}$-PVE and DPE with MAn. Gelation did not occur even at high conversions. The copolymer compositions were shown to be $1: 2 \mathrm{CH}_{3}$-PVE (or DPE)-MAn by elemental analysis.

A comparison of run 3 of Table I and runs 14 and 16 of Table II indicates that the rate of copolymerization with MAn increase in the following order: DVE $>$ PVE $>\mathrm{CH}_{3}-\mathrm{PVE}>$ DPE. This suggests that methyl substitution interfere with polymerization. The bathochromic shift of the chargetransfer absorption is in the reversed order as shown in Figure 1. Apparently, the ground-state interaction (charge-transfer absorption) of divinyl ethers and MAn is not related to the ease of copolymerization.

\section{General Structures of the Copolymers}

As discussed previously, ${ }^{6}$ the cyclocopolymer of

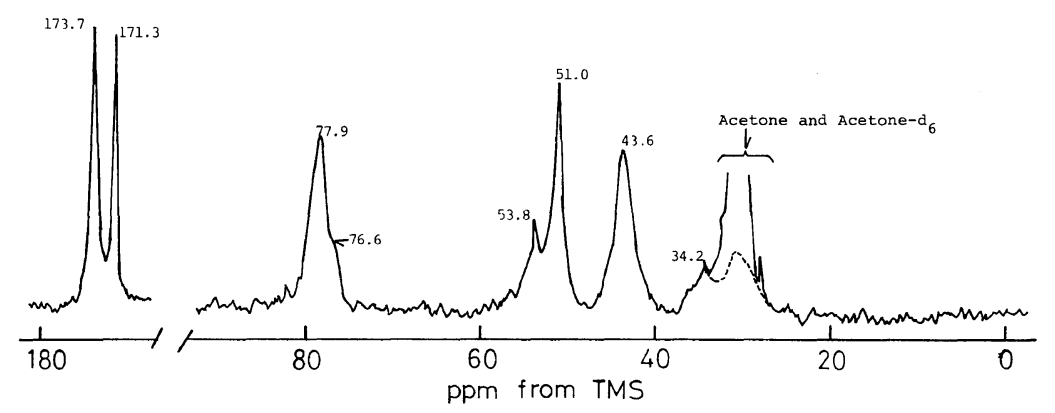

Figure 2. ${ }^{13} \mathrm{C}-\mathrm{NMR}$ spectrum of poly(DVE-MAn) obtained in $\mathrm{CHCl}_{3}$ : sample, $24 \mathrm{wt} \%$ in acetone/acetone- $d_{6}$; pulse width, $9 \mu \mathrm{s} ; 8000$ scans.

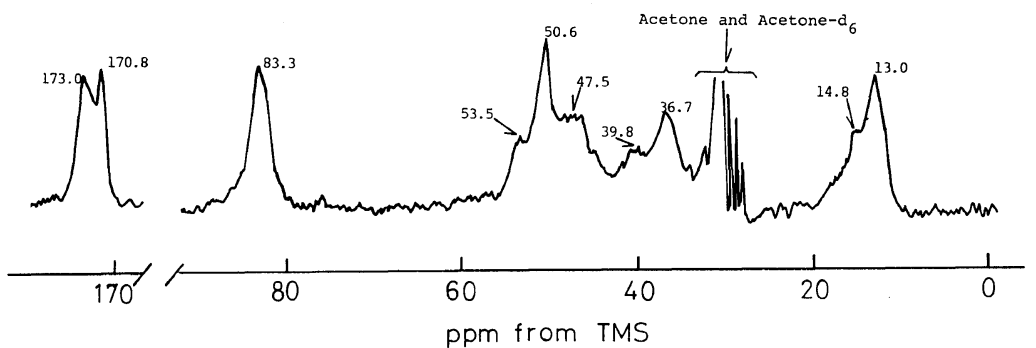

Figure 3. ${ }^{13}$ C-NMR spectrum of poly(DPE-MAn) (Table II, No. 16): sample, $19 \mathrm{wt} \%$ in acetone- $d_{6}$; pulse width, $9 \mu \mathrm{s} ; 10000$ scans.

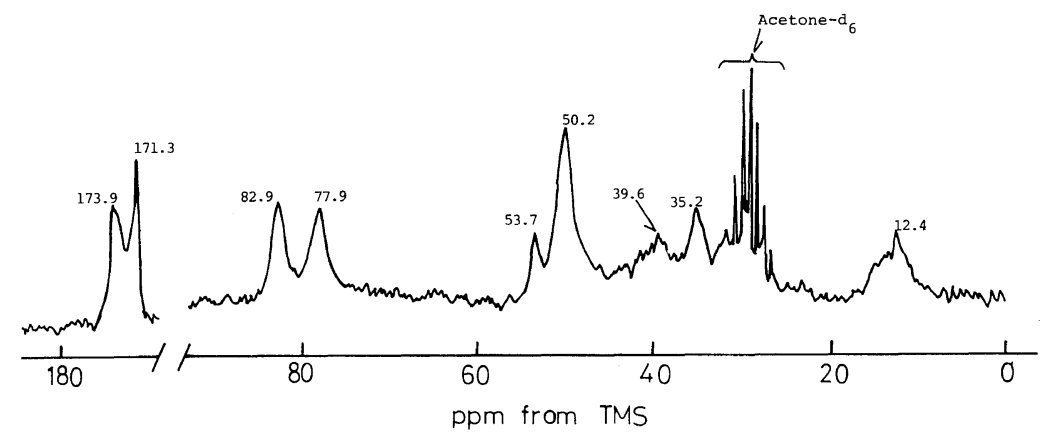

Figure 4. ${ }^{13} \mathrm{C}-\mathrm{NMR}$ spectrum of poly(PVE-MAn) (Table I, No. 10): sample, $17 \mathrm{wt} \%$ in acetone- $d_{6}$; pulse width, $9 \mu \mathrm{s} ; 10000$ scans. 


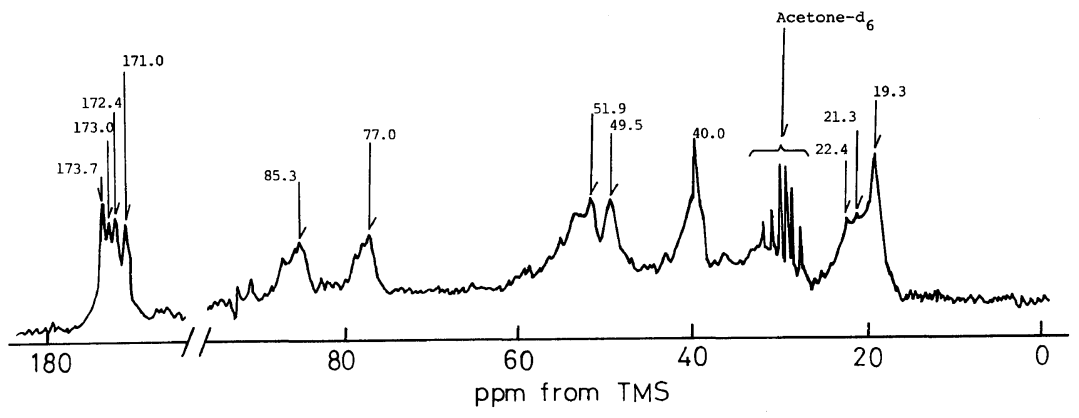

Figure 5. ${ }^{13} \mathrm{C}-\mathrm{NMR}$ spectrum of poly $\left(\mathrm{CH}_{3}-\mathrm{PVE}-\mathrm{MAn}\right.$ ) (Table II, No. 14): sample, $24 \mathrm{wt} \%$ in acetone$d_{6}$; pulse width, $9 \mu \mathrm{s}$; 6000 scans.

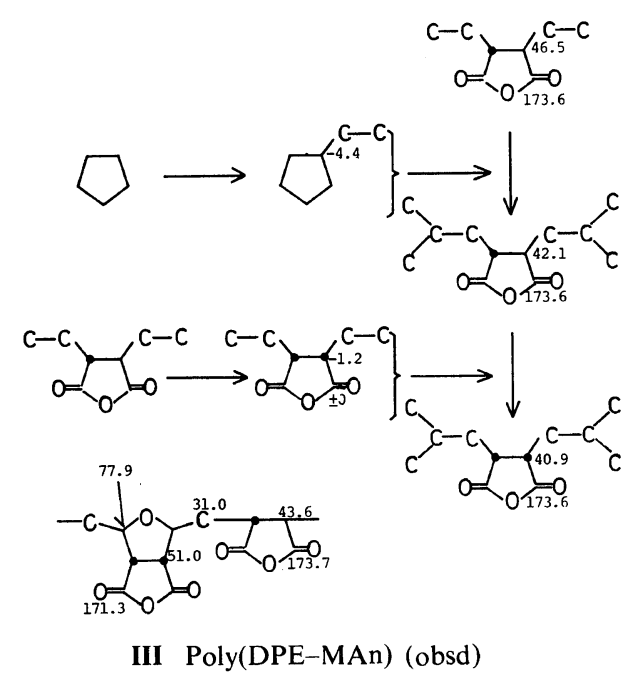

Scheme 1.

DVE and MAn is made of a symmetrical bicyclic structure (II), which gives in a ${ }^{13} \mathrm{C}-\mathrm{NMR}$ spectrum single peaks for the methine carbon adjacent to the ether oxygen and for the methine carbon of the anhydride ring. There are only two carbonyl carbon peaks considered to belong to the bicyclic and monocyclic rings (one each).

${ }^{13} \mathrm{C}-\mathrm{NMR}$ spectra of the four cyclopolymers are shown in Figures 2-5. These spectra contain common carbonyl carbon peaks at $171.0 \pm 0.3 \mathrm{ppm}$. The same chemical shift would be observed if all the copolymers contain the same bicyclic unit with the same configuration. The chemical shift of the carbonyl carbon of the monocyclic ring was recalculated by using more appropriate model compounds: cis- and trans-diethylsuccinic anhydrides. The pro- cedure is shown in Scheme 1. Improvements of $1-3$ ppm were obtained relative to the previous value. ${ }^{6}$ The calculated chemical shifts for the carbonyl carbon and the methine carbon of the trans substituted monocyclic ring, 173.6 and $42.1 \mathrm{ppm}$, respectively, are in good agreement with the observed values (173.7 and $43.6 \mathrm{ppm}$ ) given in Figure 2. The other carbonyl carbon peak at $171.0 \pm 0.3 \mathrm{ppm}$ thus belong to the bicyclic ring. In fact, the calculated shift for this carbon was $172.9 \mathrm{ppm}^{6}$ It may be concluded that the bicyclic structure is common to the four copolymers.
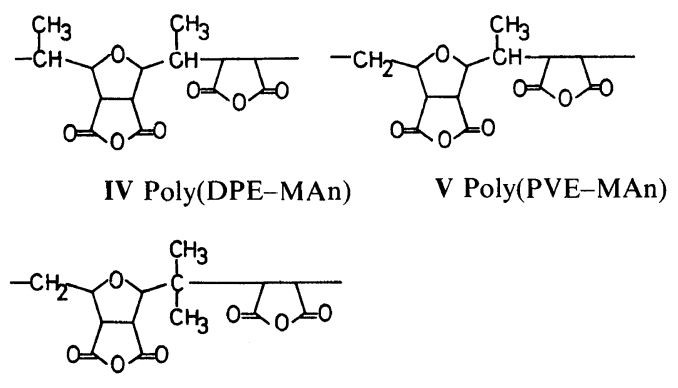

$$
\text { VI Poly }\left(\mathrm{CH}_{3}-\mathrm{PVE}-\mathrm{MAn}\right)
$$

If the bicyclization proceeds in the head-to-tail manner, the 6,5-bicyclic unit (I) will be formed, in stead of the 5,5-bicyclic unit (II) obtainable from the head-to-head bicyclization. This possibility was denied for poly(DVE-MAn) prepared in $\mathrm{CHCl}_{3}{ }^{6}$ The head-to-tail bicyclization, if it occurs, would give structure VII in the case of poly(PVE-MAn).

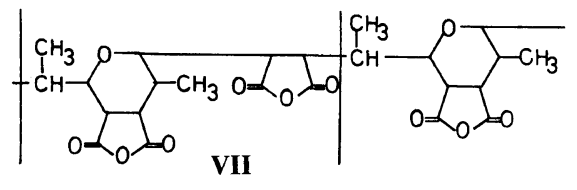




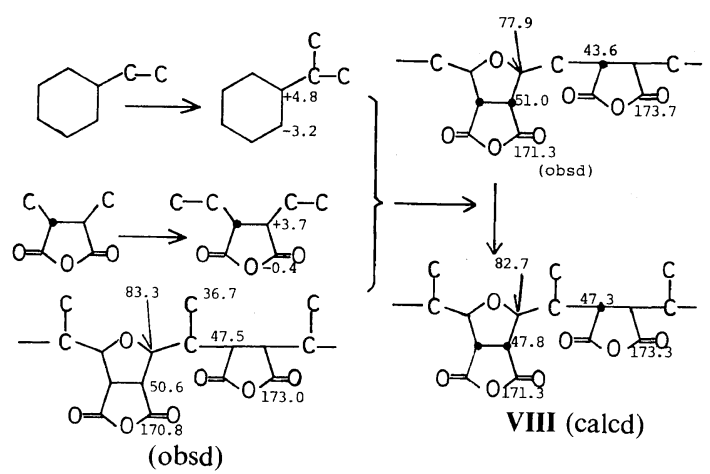

Scheme 2.

The limited number of ${ }^{13} \mathrm{C}$-NMR peaks observed for this copolymer (in particular, a single peak for the methine carbon adjacent to ether oxygen and only two carbonyl carbon peaks) indicate the formation of highly symmetrical structural units. If the pyran unit is involved in the bicyclic structure as in VII, two of the methine peaks and four of the carbonyl carbon peaks should be present.

\section{Steric Structure of Poly(DPE-MAn)}

Assuming that IV has the same configuration as that of poly(DVE-MAn), the effect of methyl substitution on the chemical shift is readily calculated using data on alkylcyclohexanes and diethylsuccinic anhydride, as shown by Scheme 2 . The calculated and observed chemical shifts agree with each other. In addition, the exocyclic methine peak in IV is located at $36.7 \mathrm{ppm}$ which is $6 \mathrm{ppm}$ apart from the exocyclic methylene peak of III ( $c a .31 \mathrm{ppm})$ and is compatible with the effect of methyl substitution.

Other types of the symmetrical bicyclic structure (IX, $\mathbf{X}$ and $\mathbf{X I}$ ) are conceivable for poly(DPE-MAn).

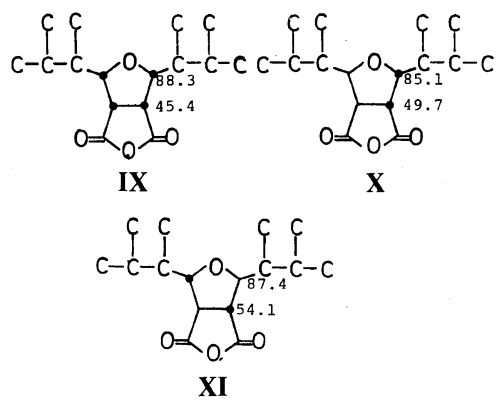

However, these structures are sterically improbable. The presence of four cis-substituents on the tetrahy- drofuran ring as in IX exerts large steric hindrance, and the trans fusion of the succinic anhydride ring and the tetrahydrofuran ring ( $\mathbf{X}$ and $\mathbf{X I})$ is sterically very difficult. Thus, structures IX and XI can be excluded by the steric consideration and disagreement of the calculated chemical shift. Structure $\mathbf{X}$ give a reasonably good agreement of the chemical shifts, but, the steric consideration renders it improbable.

The ${ }^{13} \mathrm{C}$-NMR spectrum of Figure 3 cannot be fully explained by single structure VIII. There are minor, unassigned peaks at $14.8 \mathrm{ppm}$ (methyl region), $39.8 \mathrm{ppm}$ (exocyclic methine region) and $53.5 \mathrm{ppm}$ (region of the methine carbon next to carbonyl). Similar, small peaks are present for poly(DVE-MAn) at 34.2 and $53.8 \mathrm{ppm}$ in Figure 2. In general, high-field peaks $(<55 \mathrm{ppm})$ are broad and complex in Figure 3. Poly(DPE-MAn) has many asymmetric carbons. Because of high steric hindrance, the steric variation of connection of the ring shown below may exert significant influences on the chemical shift.
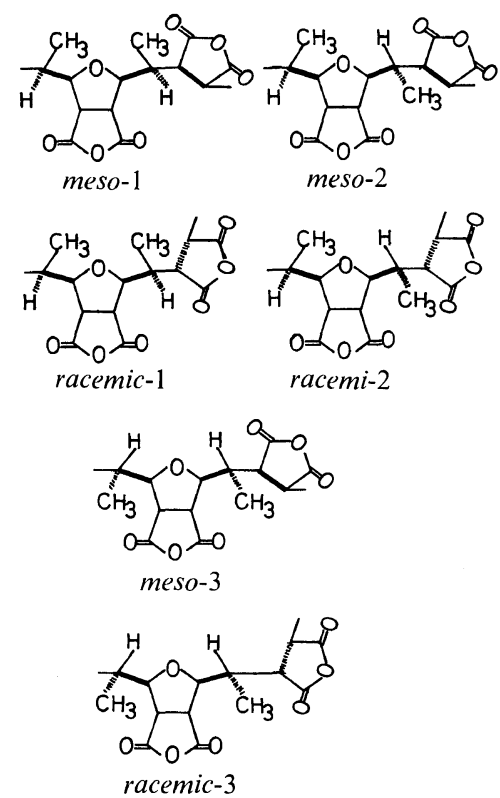

The splitting of ${ }^{13} \mathrm{C}$-NMR peaks due to the mode of connection of the unit ring has been observed for poly(divinylacetal)s $\mathbf{s}^{15}$ and poly(diallylamine)s. ${ }^{16}$

Steric Structure of Poly(PVE-MAn) and Poly( $\left.\mathrm{CH}_{3}-\mathrm{PVE}-\mathrm{MAn}\right)$

In the ${ }^{13} \mathrm{C}-\mathrm{NMR}$ spectrum of poly(PVE-MAn), 
there are twin peaks at 77.9 and $82.9 \mathrm{ppm}$. These peaks are attributed to the methine carbon adjacent to ether oxygen. The corresponding one in poly(DVE-MAn) was a single peak at $77.9 \mathrm{ppm}$. The splitting in Figure 4 is supposedly produced by the unsymmetrical methyl substitution, and therefore, stereochemistry of the bicyclic ring should be limited to one kind. It is noteworthy that the peak at $77.9 \mathrm{ppm}$ is common to the carbon peak of poly(DVE-MAn) at $77.9 \mathrm{ppm}$ and the peak at $82.9 \mathrm{ppm}$ have a counterpart at $83.3 \mathrm{ppm}$ in the spectrum of poly(DPE-MAn). This means that the bicyclic ring of $\mathbf{V}$ is formed, including stereochemistry, by combining the half bicyclic rings of II and IV; see XII.

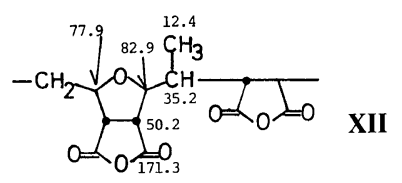

The sharp, lower-field peak of the twin peaks at $173-174 \mathrm{ppm}$ is assigned to the monocyclic carbonyl carbon in III and VIII. Broadening of the lower-field peak (173.5-173.9 ppm) in Figure 4 (XII) is understandable if a small $\gamma$-substituent effect of the methyl group on the carbonyl carbon is taken into consideration. The peak at $50.2 \mathrm{ppm}$ is common to those of Figures 2 and 3 (51.0 and $50.6 \mathrm{ppm}$, respectively) and is assignable to the junction carbon of the bicyclic ring. The pattern of peaks located in higher-fields than $45 \mathrm{ppm}$ is rather complex. This may be caused by the sterically complex mode of connection of the ring unit, as discussed above.

In the ${ }^{13} \mathrm{C}$-NMR spectrum of poly $\left(\mathrm{CH}_{3}-\mathrm{PVE}-\right.$ $\mathrm{MAn}$ ), there are relatively broad, twin peaks ( 78 and $86 \mathrm{ppm}$ ) in the region of the methine carbon next to ether oxygen. The peak at $78 \mathrm{ppm}$ corresponds almost exactly to the methine carbon peak (77.9 ppm) of poly(DVE-MAn). The chemical shift difference between the twin peaks (85.3$77.0=8.3 \mathrm{ppm}$ at the peak maximum) is close to the effect of gem-dimethyl substituent on the $\beta$ carbon. $^{17}$

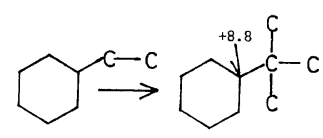

Polymer J., Vol. 13, No. 7, 1981
The splitting of the carbonyl carbon peak into four may be explained by the gem-dimethyl effect, as discussed for poly(PVE-MAn). It is thus suggested that the polymer contains unique monocyclic and bicyclic units XIII as in the case of the other copolymers. Tentative assignments are given in XIII.

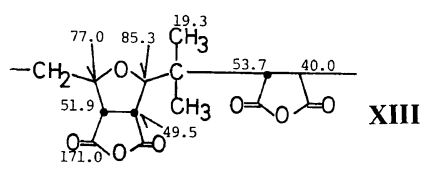

The higher-field peaks are generally broad. This may be caused again by restriction of the polymer conformation.

\section{Mode of Propagation}

It is assumed in the above discussion that the site of the first reaction remains the same (i.e., less substituted vinyl group) in the unsymmetrical divinyl ethers. Then, structures II- $\mathbf{V}$ are formed. If, however, the mode of propagation is not constant with PVE and $\mathrm{CH}_{3}-\mathrm{PVE}$, the following structures may also be involved.
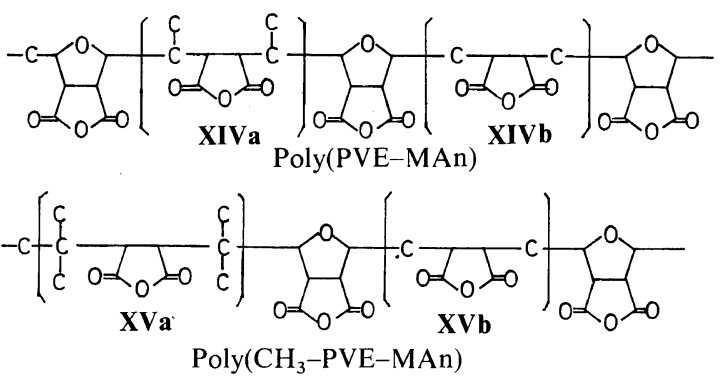

This poly-PVE-MAn) contains two monocyclic units (XIVa and XIVb) in addition to the normal monocyclic unit in V. Structure XIVa is the same as the monocyclic unit of poly(DPE-MAn), IV, and structure XIVb is the same as the monocyclic unit of poly(DVE-MAn), II. Therefore, the presence of these irregular structures can be confirmed or denied by comparing ${ }^{13} \mathrm{C}-\mathrm{NMR}$ spectra of the copolymers. If XIVa is present, peaks characteristic of the monocyclic unit of poly(DPE-MAn) must be found. The peaks characteristic of the monocyclic unit in VIII (Scheme 2) cannot be found in the spectrum of Figure 4. Similarly, the peaks characteristic of XIVb ( $c f$. Figure 2) cannot be found in 


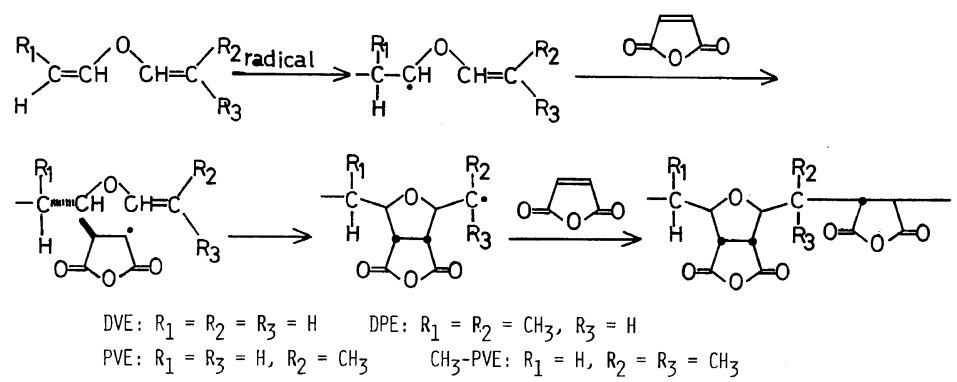

Scheme 3.

Figure 4. These results indicate that the irregular structures cannot be present in poly(PVE-MAn) at least as major components.

Similar arguments can be applied to possible irregular structures in poly $\left(\mathrm{CH}_{3}-\mathrm{PVE}-\mathrm{MAn}\right)$, and a comparison of Figure 5 with Figure 2 indicates their absence.

\section{Course of Cyclocopolymerization}

Scheme 3 summarizes the copolymerization process of divinyl ether and its methyl derivatives with maleic anhydride. The major course of propagation is the same among the four copolymerization systems. The divinyl monomers always react with the propagating radical at the less substituted vinyl group. The resulting radical adds to maleic anhydride and the new propagating species cyclizes in a head-to-head manner to form the $[3,3,0]$ bicyclic ring. The cyclization includes the trans ring closure with formation of the cis bicyclic junction. The cyclized radical then adds to maleic anhydride. The double bond of maleic anhydride opens in the trans fashion in the subsequent process. Although the polymerization rate and molecular weight are lowered with methyl substitution, the pattern of the propagation remains the same.

\section{REFERENCES}

1. I. Iliev, M. Georgieva, and V. Kabaivanov, Uspechi Chimmii, 43, 135 (1974).

2. D. S. Breslow, E. I. Edwards, and N. R. Newburg, Nature, 246, 160 (1973).

3. G. B. Butler, J. Polym. Sci., 48, 279 (1960); J. Macromol. Sci., Chem., 5, 219 (1971).

4. G. B. Butler and Y. C. Chu, J. Polym. Sci., Polym. Chem. Ed., 17, 859 (1979).

5. R. J. Samuels, Polymer, 18, 452 (1977).

6. T. Kunitake and M. Tsukino, J. Polym. Sci., Polym. Chem. Ed., 17, 877 (1979).

7. M. Tsukino and T. Kunitake, Macromolecules, 12, 387 (1979).

8. M. Tsukino and T. Kunitake, Polym. J., 13, 657 (1981).

9. G. M. Bennett and W. G. Philip, J. Chem. Soc., 1930 (1928).

10. C. C. Price and W. H. Snyder, Tetrahedron Lett., 69 (1962).

11. J. Bode and H. Brockmann, Jr., Chem. Ber., 105, 34 (1972).

12. K. Nagarajan, Ch. Weissmann, H. Schmid, and P. Karrer, Helv. Chim. Acta, 46, 1212 (1963).

13. H. Wren and J. W. E. Haller, J. Chem. Soc., 230 (1937).

14. G. B. Butler and K. Fujimori, J. Macromol. Sci., Chem., A6, 1533 (1972).

15. M. Tsukino and T. Kunitake, Polym. J., 11, 437 (1979).

16. S. R. Johns, R. I. Willing, S. Middleton, and A. K. Ong, J. Macromol. Sci., Chem., A10, 875 (1976).

17. J. Q. Adams and L. P. Lindeman, Prepr. Am. Chem. Soc., Div. Petrol. Chem., 17, C4 (1972). 